\title{
Complete Absence of The Left Pericardium in On-Pump Coronary Artery Bypass Operation
}

\section{Atilla Orhan}

Selçuk University Faculty of Medicine, Department of Cardiovascular Surgery, Konya, Turkey

\begin{abstract}
The complete absence of the left pericardium is a rare condition, which is found incidentally during surgical procedures. Preoperative computerized tomography imaging is not a routine practice in open heart surgery, and the diagnosis of these cases is often overlooked. In a patient
\end{abstract}

undergoing on-pump coronary artery bypass surgery, we present a rare case of a left pericardium after sternotomy.

Keywords: Pericardium, congenital heart defect, coronary artery bypass grafting

Meeting presentation: The manuscript was presented as a poster presentation 14th International Congress of Update in Cardiology and Cardiovascular Surgery from April 5th to $8^{\text {th }}, 2018$ in Antalya, Turkey.

Orhan A.: Complete Absence of The Left Pericardium in On-Pump Coronary Artery Bypass Operation

EJCM 2018; 06 (4): 157-160. DOI: 10.32596/ejcm.00025

Address for Correspondence: Atilla Orhan, Selçuk University Faculty of Medicine, Department of Cardiovascular Surgery, Konya, Turkey

e-mail: atillaorhan@gmail.com

Received: 26.05.2018 Accepted: 03.06.2018 Published: 30.11.2018

${ }^{\circ}$ Copyright 2018 by Heart and Health Foundation of Turkey (TÜSAV) / E Journal of Cardiovascular Medicine published by Galenos Publishing House. 


\section{Introduction}

The complete absence of the left pericardium (CALP) is a rare condition, which is incidentally found during surgical procedures. ${ }^{(1)}$ Because the preoperative computerized tomography (CT) imaging is not a routine practice in open heart surgery interventions, the diagnosis of such cases is often overlooked.

We present a rare case of the CALP after sternotomy in a patient undergoing on-pump coronary artery bypass surgery.

\section{Case}

A 61-year-old Central Anatolian gentleman was referred to our clinic because of his coronary artery disease requiring a coronary artery bypass. A coronary artery angiogram revealed 3-vessels coronary artery

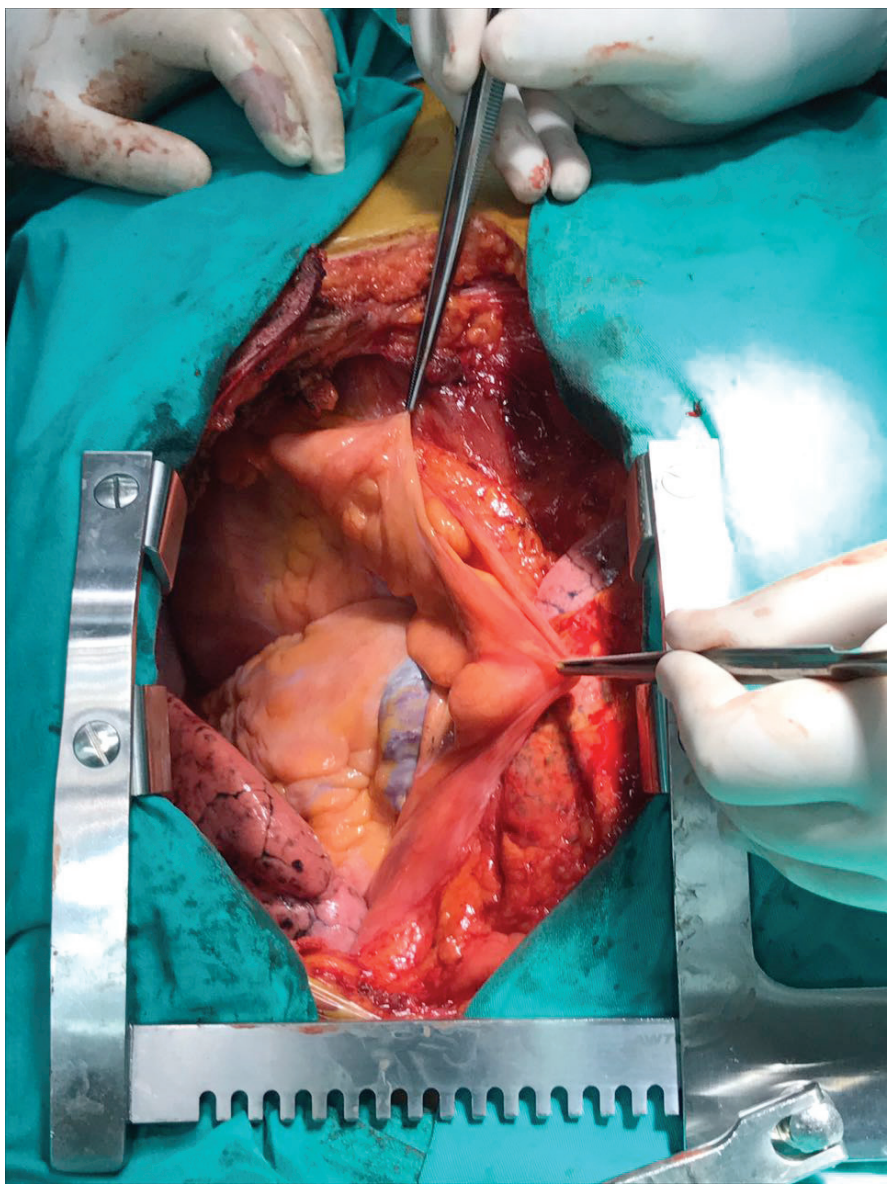

Figure 1. Intraoperative view disease requiring open heart surgery. Preoperative chest $\mathrm{X}$-ray, electrocardiography, and echocardiography showed no abnormal findings. The patient was operated under elective conditions. At the beginning of the operation, we recognized the complete defect of the left pericardium (Figure 1). On intraoperative view, the left pericardial defect extended from the front of the left ventricle to the left lung hilum. The existing pericardium covered only a small area of the right ventricule. Other sides of the heart, the pericardium was not visualized. The left phrenic nerve lied down in the fat tissue between the heart and the lung (Figure 2). The postoperative period of the patient was uneventful and the patient was discharged. We retrospectively searched the hospital's archive records and found a chest $\mathrm{CT}$ performed one year ago. The chest $\mathrm{CT}$ report was normal. When $\mathrm{CT}$ was reevaluated in the

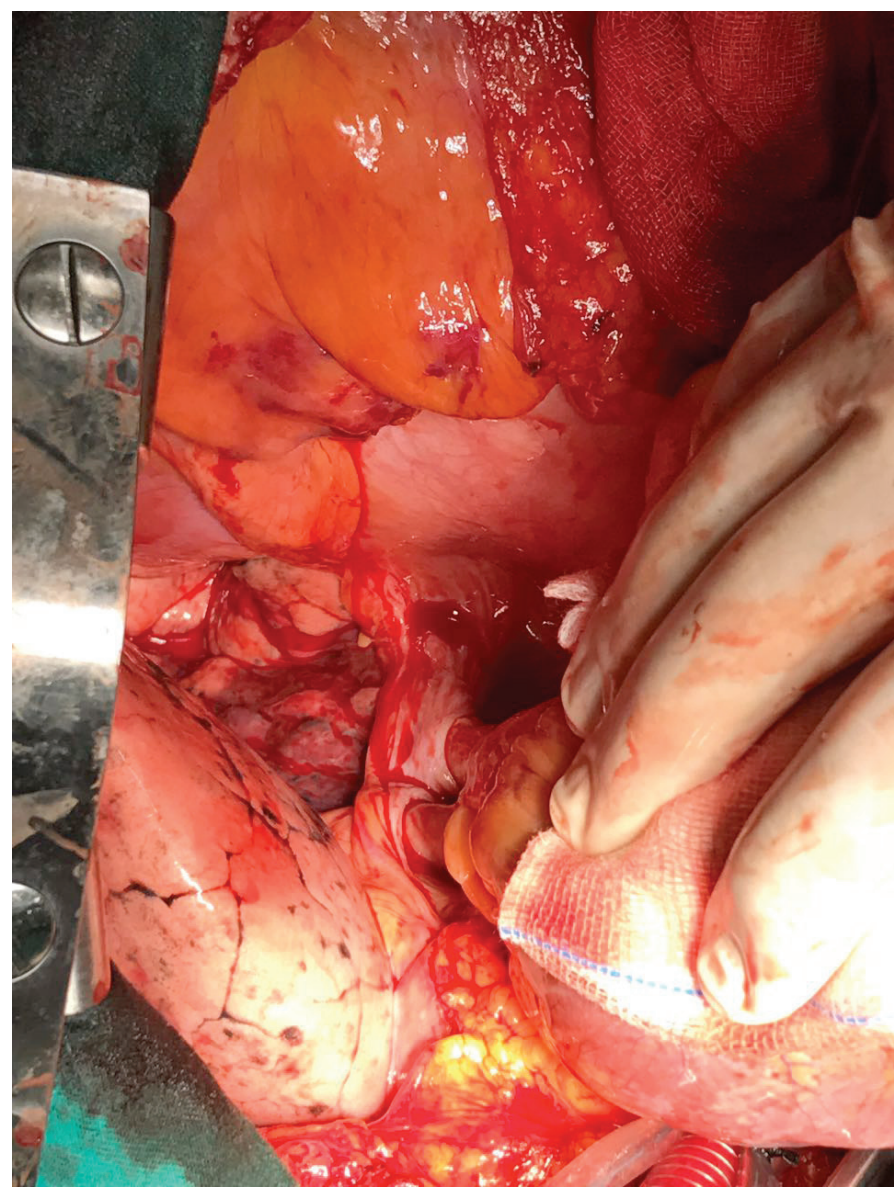

Figure 2. Intraoperative view 
direction of our operation findings, it was seen that the heart was completely in the left chest and there was a flattening of the axis of the heart (Figure 3).

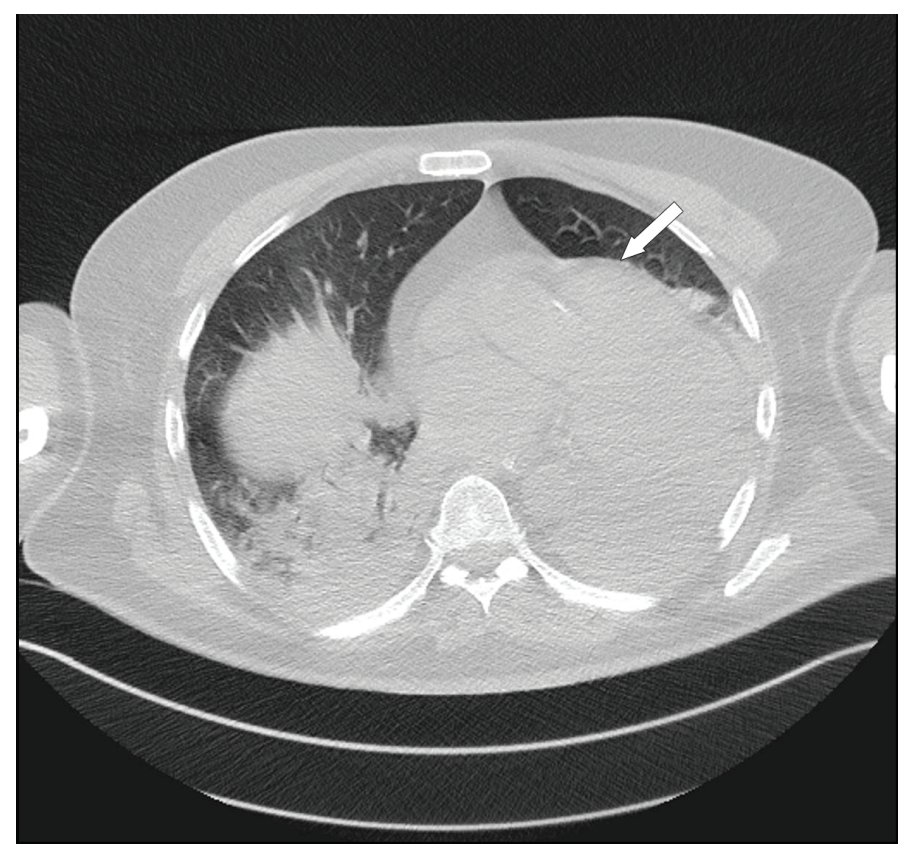

Figure 3. The chest computerized tomography section clearly shows the absence of pericardium around the left ventricle and the leftward turn of the heart (arrow)

\section{Discussion}

The CALP is infrequent. ${ }^{(1)}$ The number of cases reported in the literature is less than 500 up to now (2). It is three times more common in men compared to women. (2) It is an anomaly caused by the incorrect division of pleuro-pericardial space at the $5^{\text {th }}$ week of embryonic life.

(3) The CALP anomaly may exist by itself or may co-exist with other cardiac or pulmonary anomalies. ${ }^{(4)}$ A CALP is classified as complete or partial and can be left or right depending on its location. ${ }^{(5)}$

The CALP doesn't have specific symptoms, and most of them are noticed by incidentally or during surgery. ${ }^{(1)}$ Non-specific findings of the CALP include paroxysmal chest pain and dyspnea. ${ }^{(6)}$ There was a typical chest pain due to coronary artery disease and no evidence was suggesting CALP in our case.
The CALP causes lung tissue to enter between the aorta and the main segment of the pulmonary artery as in our case. ${ }^{(4)}$

Diagnosis is difficult because of the rare occurrence of these cases. ${ }^{(4)}$ In addition, most clinicians and radiologists have insufficient experience with these cases. Most of the CALP cases stay unnoticed because of this insufficient experience. ${ }^{(5)}$

Although routine examinations such as chest X-ray, electrocardiogram (ECG) and echocardiograpy may help to detect the absence of the pericardium, these tests do not provide specific findings. The ECG may indicate right axis deviation in the horizontal plane, incomplete or complete $\mathrm{RBBB}$ and poor $\mathrm{R}$ wave progression. The Chest $\mathrm{X}$-ray may show levoposition of the heart. Finally, echocardiography exam may show abnormal heart position and movement. (4) But these findings are often absent as it is in our case.

Preoperative thorax CT and cardiac magnetic resonance (MR) are not routine procedures but may give valuable findings such as defect size, herniation, and other pathologies. ${ }^{(3,4)}$ We investigated patient's record retrospectively and reevaluated a Thorax CT performed 2 years ago. Despite it have been reported normal, it was clear that the heart was deviating to the left side and the lung tissue was interposed between the aorta and the main pulmonary artery.

In cases of a suspicion of the absence of the pericardium, cardiac MR is a preferred test, especially for CALPs. Cardiac MR describes the anatomical relationship between the heart, the mediastinum, and the lung with high soft tissue contrast and multi-plane capability. However, the diagnosis of a pericardial defect is not even clear in cardiac MR. ${ }^{(4)}$

Since there are no specific findings in $\mathrm{CT}$ or MR, rare cases such as the absence of pericardium are frequently missed by radiologists.

The absence of pericardium did not cause additional difficulty in our operating procedure. We could not put retraction sutures on the left side. We lifted heart's apex for 
obtuse marginal branches of the left circumflex coronary artery anastomosis. Nevertheless, when performing a sternotomy in the absence of the pericardium, additional care must be taken for possible retrosternal adhesions. During the surgery, we use the pericardium to position the heart. In the CALP cases, additional methods can be used to position the heart (e.g., holding the apex of the heart still with a pad in the hands of a second assistant during bypass, or with the aid of a stabilizer). In general, this is the main difference between the cases with- and withoutthe pericardium. We have not performed any repair on the pericardium because of the large defect and the nature of open heart surgery. However, small and partial defects should be repaired, especially due to the risk of kinking in bypass grafts. ${ }^{(1)}$ It is also necessary to adjust the size and placement of grafts in small defects. ${ }^{(1)}$

\section{Conclusion}

Although the absence of pericardium is an incidental finding, it should be kept in mind when there are nonspecific chest complaints. Developments in the diagnostic imaging techniques may help the recognition of congenital pericardial absence. Radiologists' awareness and identification of characteristic radiological findings are important in preventing complications. When such defects are encountered during open heart surgery, it is important to recognize the different anatomy and to change the surgical technique accordingly. It is also necessary to adjust the size and location of the grafts in the small defects.

Disclosure and conflicts of interest: The authors declare no conflict of interest.

\section{References}

1. Reddy CS, Ali SN, Raamaanuhadaasudu CK, Parimi SS, Reddy TR, Reddy VK. Off-pump coronary artery bypass in congenital absence of pericardium. Asian Cardiovasc Thorac Ann 2013;21:473-5.

2. Juarez AL, Akerstrom F, Alguacil AM, Gonzalez BS. Congenital partial absence of the pericardium in a young man with atypical chest pain. World J Cardiol 2013;5:12-4.

3. Koo CW, Newburg A. Congenital absence of the right pericardium: embryology and imaging. J Clin Imaging Sci 2015;5:12.

4. Bueno Palomino A, Palomar Estrada A, Crespin Crespin M, Garcia Fuertes D. Congenital complete absence of pericardium in a young woman with non-specific symptoms. Rev Port Cardiol 2014;33:249.

5. Lopez D, Asher CR. Congenital Absence of the Pericardium. Prog Cardiovasc Dis 2017;59:398-406.

6. Topilsky Y, Tabatabaei N, Freeman WK, Saleh HK, Villarraga HR, Mulvagh SL. Images in cardiovascular medicine. Pendulum heart in congenital absence of the pericardium. Circulation 2010;121:1272-4. 